\title{
Control of the induction soldering on the basis of process temperature indirect measurements
}

\author{
Vadim S. Tynchenko ${ }^{1, *}$, Vyacheslav E. Petrenko ${ }^{1}$, and Alexander V. Murygin ${ }^{1}$ \\ ${ }^{1}$ Reshetnev Siberian State University of Science and Technology, Department of Information Control \\ Systems, 31 Krasnoyarsky Rabochy Av., Krasnoyarsk, Russian Federation
}

\begin{abstract}
The article suggests the use of indirect measurements to control the process of induction soldering. Based on the equations of thermodynamics, it is proposed a scheme for approximating the temperature values in the solder zone on the basis of information from pyrometric sensors aimed at an area remote from the soldered joint site. A model-algorithmic instrument for indirect temperature measurements in the soldering technological process is developed, the scheme of which is presented in the article. The software of waveguide paths induction soldering control in the form of an already existing system module has been developed, which allows not only to carry out experimental studies on control algorithms, but also to implement a full-scale experiment, the results of which confirm the effectiveness of the proposed approach.
\end{abstract}

\section{Introduction}

In the aerospace industry, rigid restrictions on the mass of aircraft cause the use of various elements of payload modules with optimal mass-size characteristics. This also applies to waveguide paths of antenna-feeder devices of space vehicles. At the same time, the reliability of the elements must be high enough to ensure their functioning during the whole period of active existence, which for modern space communication apparatus is 12-15 years. [1]

Various types of electromagnetic waves can propagate along the waveguide. The predominant propagation of waves of one type or another depends on the geometric dimensions of the waveguide, the frequency of the excited electromagnetic oscillations, and the method of their excitation. [2, 3]

The component elements of the waveguide paths are assembled by induction soldering, which has a number of undeniable advantages with respect to other types of heating, such as open flame heating or heating in an oven.

In the process of induction heating control, when forming soldered joints of waveguide paths, it is required to measure the process temperature, for which the use of pyrometric sensors is best suited $[3,4]$. In the process of melting flux, covering the place of the brazed connection in a significant way, the emissivity of the surface of the workpiece changes, which complicates the process of induction heating control depending on the temperature

* Corresponding author: vadimond@mail.ru 
information from the solder zone [5]. Thus, it is expedient and relevant to use in the information control system indirect temperature measurements which are made at a distance spot from the solder zone.

\section{Scheme of the induction soldering process control}

In studies $[6,7]$ a complex of automated equipment for controlling the technological process of waveguide paths induction soldering is proposed. For control, it is proposed to use information from two pyrometric sensors aimed at the assembled elements.

The layout of the workpiece in the inductor, as well as the scheme for targeting noncontact pyrometric temperature sensors, are shown in Fig. 1.

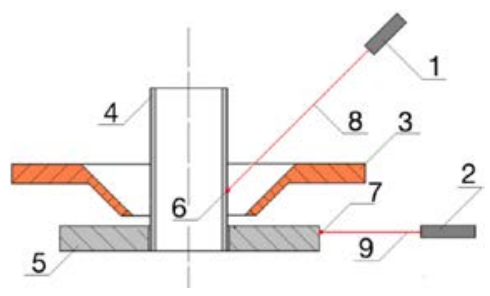

Fig. 1. Pyrometers targeting scheme.

However, using this arrangement of pyrometric sensors, the following effect appears: the measurement of the pyrometer aimed at the solder zone is significantly distorted during the melting phase of the flux and its evaporation due to a change in the emissivity of the waveguide assembly material.

This limits the possibility of direct temperature control in the area of elements joint, which is due to the following factors:

- In the soldering zone, there is solder,

- The area closest to the soldering zone is covered with flux,

- The size of the inductor window limits the view of the pyrometer in the solder zone.

In a number of cases, this discrepancy between the measured temperature and the actual one does not allow achieving the desired repeatability of the soldering technological process, which leads to an increase of the manufacturing defects. Elimination of this disadvantage is possible by compensating of distortions introduced by the flux as when the pyrometer spot is intentionally placed on the already covered surface with flux, or as a result of its spreading over the surface of the product as a result of melting during the technological process. For this it is proposed to perform indirect temperature measurements obtained from the clean surface of the product at a certain distance from the soldering zone, which is possible with the application of calculations for thermal models.

Thus, the implementation of the control in automated system based on such measurements is difficult and not accurate without additional compensation. If the pyrometer is removed from the soldering zone, the reliability of the information decreases and it becomes necessary to compensate for this unreliability of temperature measurements.

\section{Induction soldering control based on indirect measurements}

In order to eliminate the above-described effect in the measurements, it is proposed to use indirect measurements of the process temperature in the soldering zone, realized as follows: 1) The pyrometer is aimed at the clean area of one of the soldered elements not covered with flux, 
2) Using the mathematical apparatus of thermal processes (thermodynamics), an evaluation of the elements heating temperature in the soldering zone is made,

3) The calculation of the control action in an automated system based on indirect measurements is made.

\subsection{Implementing calculations of indirect measurements}

To implement indirect measurements, the use of the mathematical apparatus of the welding processes theory is proposed. In order to simplify the calculation of the temperature in the soldering zone, a number of the following assumptions are made on the basis of indirect measurement of temperature [8]:

- Waveguide pipe is considered as an infinite rod,

- The current induced in the workpiece at a certain point in time creates an elementary heat source at a point perpendicular to the inductor window in its part as close as possible to the waveguide tube, and also uniform in its cross section.

Thus, using the thermal model of heating an infinite rod of continuously operating heat sources, it is possible to realize an approximate calculation of the temperature in the solder zone. Based on the measurements obtained from the pyrometer, whose field of view is outlaid on some distance from the soldering zone, and also monitoring the current voltage information on the induction generator, the initial position of the inductor window relative to the workpiece, and its variation during the soldering process, it is possible to correct the indirect temperature measurement by formula (1), thereby obtaining for control the temperature data in the soldering zone.

$$
\begin{aligned}
& T_{\text {zone }}\left(x_{\text {pyro }}, x_{0}, \Delta x, t, q\right)=T_{\text {mes }}\left(\mathrm{t}-\mathrm{t}^{\prime}\right)+ \\
& +\int_{t-t^{\prime}}^{t} \frac{q\left(t-t^{\prime}\right)}{F \cdot c p \cdot \sqrt{4 \pi a\left(t-t^{\prime}\right)}} \exp \left(-\frac{\left(x_{\text {pyro }}-\left(x_{0}+\Delta x\right)\right)^{2}}{4 a\left(t-t^{\prime}\right)}-b\left(t-t^{\prime}\right)\right) d t^{\prime}
\end{aligned}
$$

where $T_{\text {mes }}$ - the temperature measured by the pyrometer at the current time $\left({ }^{0} \mathrm{C}\right) ; q\left(t-t^{\prime}\right)-$ the amount of heat determined by the current inductor current $(\mathrm{J}) ; F$ - pipe section $\left(\mathrm{m}^{2}\right) ; c p$ - volume heat capacity $\left(\mathrm{J} / \mathrm{m}^{3}\right) ; a$ - coefficient of thermal conductivity; $x_{\text {pyro }}$ - distance from the soldering zone to the pyrometer spot $(\mathrm{m}) ; x_{0}$ - initial position of the heating source, relative to the solder zone $(\mathrm{m}) ; \Delta x$ - change in the position of the heating source, relative to the initial (m); $t$ - time (sec.); $b$ - coefficient of heat transfer to the external environment from the surface; $t^{\prime}$ - the time of an instantaneous heat source action occurrence (sec.).

To study the effectiveness of the proposed mathematical apparatus, a series of experiments was carried out with the following arrangement of the pyrometers (Fig. 2).

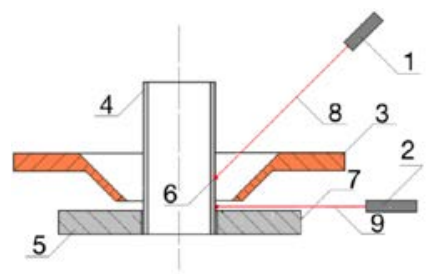

Fig. 2. Pyrometers targeting scheme to study the effectiveness of the indirect temperature measurements.

According to the results of the experiments series, it was revealed that the proposed mathematical apparatus allows to approximate the temperature in the soldering zone by performing indirect measurements.

A typical graph obtained in the course of experimental studies is shown in Fig. 3. 


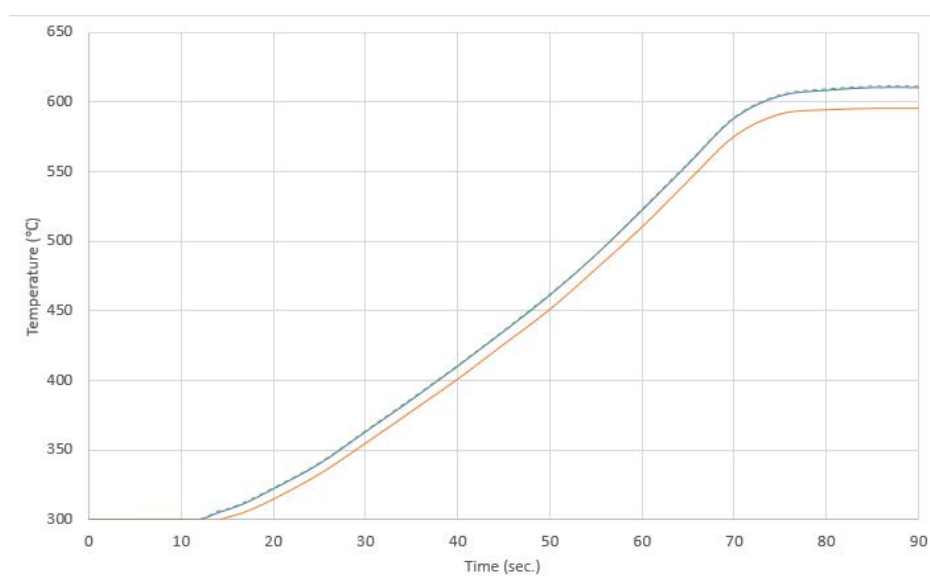

Fig. 3. Heating diagram at points of a waveguide pipe without flux: blue graph - temperature in the soldering zone; red graph - temperature in the distance from the soldering zone by $2 \mathrm{~cm}$; green shaded graph - the calculated value of the temperature in the solder zone on the basis of indirect measurements.

As a result of calculations for 25 experiments, the root-mean-square error was $0.21{ }^{\circ} \mathrm{C}$, which is permissible within the technological process of induction soldering.

\subsection{Soldering process control scheme}

Using the RAD Studio programming system, an automated induction soldering control system based on indirect measurements was developed. The system uses two control loops: 1) The control loop for controlling the heating rate according to the temperature of the waveguide pipe.

2) Control of the workpiece position relative to the inductor on the basis of a comparison of the welded elements temperatures.

The block diagram of the developed automated system is shown in Fig. 4.

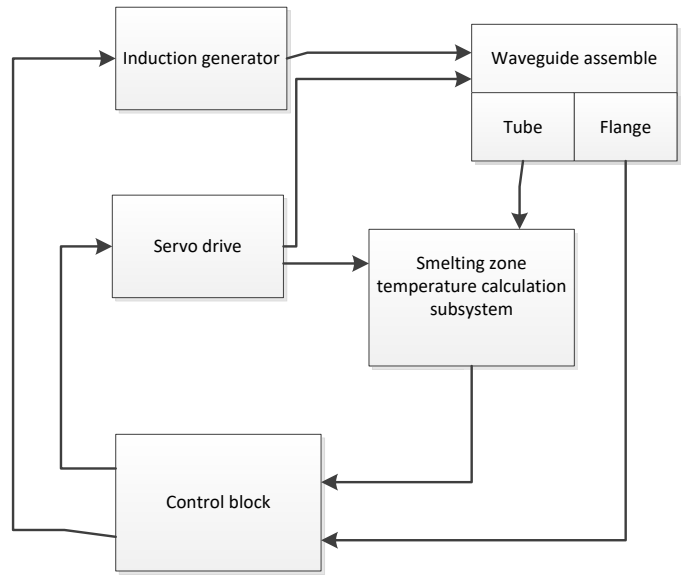

Fig. 4. Block diagram of a two-circuit induction soldering control system.

With the use of the developed system, a series of experiments was carried out both on adjusting the parameters of the control algorithms and on the possibility of using indirect measurements of the waveguide paths soldered elements temperature. 


\section{Experimental study}

To conduct experimental studies, soldering of waveguide assemblies of sizes $58 \times 25 \mathrm{~mm}$ was carried out. A typical heating curve during the soldering process is shown in Fig. 5.

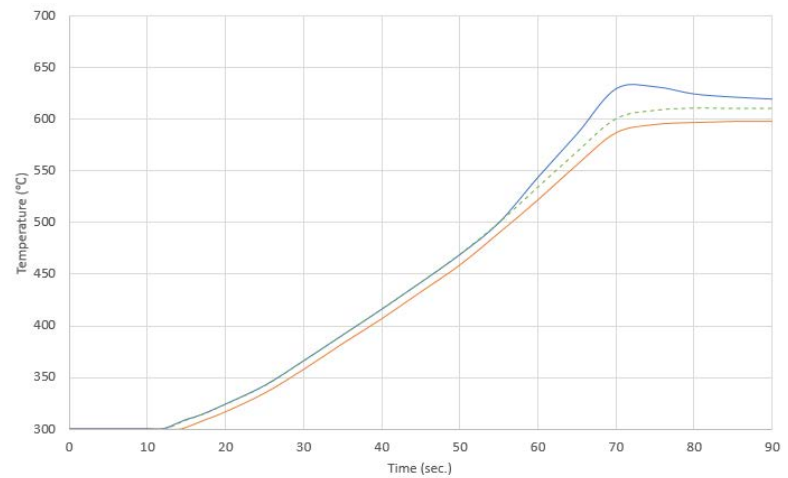

Fig. 5. Heating diagram of a waveguide elements when conducting experiments: blue graph temperature in the soldering zone; red graph - temperature in the distance from the soldering zone by $2 \mathrm{~cm}$; green shaded graph - the calculated value of the temperature in the solder zone on the basis of indirect measurements.

As can be seen from the graph, the readings of the pyrometer aimed at the solder zone (blue curve) have significant distortions due to flux melting and changes in the emissivity of the measured surface in the soldering zone. The implementation of the control based on the such information does not allow to carry out high-quality solder joint with high repeatability of the technological process. The distortions introduced by the flux are random in consequence of the different thicknesses of the applied flux layer.

The application for controlling indirect measurements makes it possible to organize efficient control of the technological process with a high degree of repeatability. The disadvantage of the proposed method is the need for the operator to accurately indicate the mass-dimensional characteristics of the waveguide path welded elements, however, taking into account the large-scale production at the enterprises of the rocket and space industry, such a disadvantage can be considered insignificant.

\section{Conclusion}

The article describes in detail the technological process of waveguide paths soldering using induction heating, as well as the main problems associated with its use. A new scheme for the location of non-contact pyrometric sensors is proposed, as well as a procedure for calculating the temperature of the soldering process based on indirect measurements.

The proposed method of compensation of distortions introduced by flux as a result of the soldering process was tested: using a developed algorithmic and software, a series of full-scale experiments was performed, the results of which confirm the effectiveness of the proposed approach. Application of the research results will reduce the amount of rejects in the production of waveguide tracts, improve the efficiency of the soldering process by reducing the human factor and, accordingly, bring significant economic benefits to the rocket and space industry.

The reported study was funded by the President of the Russian Federation grant for state support of young Russian scientists No MK-6356.2018.8. 


\section{References}

1. P. Cicconi, A.C. Russo, M. Germani, M. Prist, E. Pallotta, A. Monteriu, Cyberphysical system integration for industry 4.0: Modelling and simulation of an induction heating process for aluminium-steel molds in footwear soles manufacturing $3 \mathrm{rd}$ International Forum on Research and Technologies for Society and Industry (RTSI 2017) 1 1-8 (2017)

2. I. Makulov, A. Alitdinov, The equipment and application features of induction heating in oil and gas industry Int. Conf. on Ind. Eng., App. and Man. (ICIEAM 2107) 1 1-4 (2017)

3. J. Sun, S. Li, C. Qiu, Y. Peng Numerical and experimental investigation of induction heating process of heavy cylinder Applied Thermal Engineering 134 341-352 (2018)

4. E. Mani, T. Udhayakumar, Effect of prior austenitic grain size and tempering temperature on the energy absorption characteristics of low alloy quenched and tempered steels Mater. Sci. Eng. A 716 92-98 (2018)

5. L. Pan, P. Li, X. Hao, J. Zhou, H. Dong Inhomogeneity of microstructure and mechanical properties in radial direction of aluminum/copper friction welded joints Journal of Mat. Proc. Technology 255 308-318 (2018)

6. A.V. Murygin, V.S. Tynchenko, V.D. Laptenok, O.A. Emilova, A.N. Bocharov Complex of automated equipment and technologies for waveguides soldering using induction heating IOP Conf. Series: Mat. Science and Eng. 173012023 (2017)

7. V.S. Tynchenko, A.V. Murygin, V.E. Petrenko, Yu.N. Seregin, O.A. Emilova A control algorithm for waveguide path induction soldering with product positioning IOP Conf. Series: Mat. Science and Eng. 255012018 (2017)

8. N.N. Rykalin, Calculations of thermal processes in welding Fizmatlit 296 (1951) 